\title{
LA MUJER EN LAS PROFESIONES DE LA SALUD
}

\section{Laura Rueda C.*}

Resumen: El presente artículo busca establecer, a partir de la detección de competencias afectivas entre las jóvenes que se encuentran estudiando profesiones de la salud, una reflexión acerca de la presencia femenina en esta área profesional. El análisis incluye elementos del desarrollo moral femenino, de la vocación por cuidar y prevenir el riesgo, y del perfil profesional que se va generando en las carreras dedicadas al cuidado de la salud humana.

Palabras clave: desarrollo moral, ética del cuidado, profesiones de la salud

\section{THE WOMAN IN THE HEALTH CARE PROFESSIONS}

Abstract: This paper tries to establish, through identifying the affective capacities of young women who are studying health care professions, a reflection about the feminine presence in this professional sector. This analysis includes some aspects of the feminine moral development, the vocation to care and to prevent risks and the professional profile that has been developed in careers dedicated to health care.

Key words: moral development, ethics of care, health care professions

\section{A MULHER NAS PROFISSÕES DE SAÚDE}

Resumo: O presente artigo procura estabelecer, a partir da detecção de competencias afetivas entre as jovens estudantes nas profissões de saúde, uma reflexão sobre a presença feminina nesta área profissional. A análise inclui elementos do desenvolvimento moral feminino, da vocação de cuidar e previnir o risco, bem como do perfil profissional que vai sendo gerado nas profissionais dedicadas ao cuidado da saúde humana.

Palavras chave: desenvolvimento moral, ética do cuidado, profissões da saúde

* Terapeuta Ocupacional, Magíster en Bioética. Profesora Asistente de la Facultad de Medicina y colaboradora del Centro Interdisciplinario de Estudios en Bioética, Universidad de Chile. Chile

Correspondencia: ruedal@chi.ops-oms.org 


\section{Introducción}

Desde el surgimiento de la medicina occidental, cuyo exponente más reconocido es el médico griego Hipócrates de Cos, se define el ejercicio profesional dirigido al cuidado $\mathrm{y}$ restablecimiento de la salud de las personas. Es necesario acotar que las acciones de este médico originario reunían todas las funciones que en la actualidad desarrollan las diferentes profesiones de la salud.

En aquella época las mujeres no accedían a la educación formal para adquirir las competencias. Sin embargo, los cuidados y acciones dirigidas a procurar el bienestar del enfermo coinciden con los quehaceres femeninos domésticos. La siguiente cita del primer aforismo hipocrático transmite esta observación(1):

Vida breve y arte largo:

Ocasión acelerada

Experimento arriesgado.

Juicio difícil. No basta

Que cumpla con sus deberes

El profesor de más fama;

Es necesario concurran

Otras cosas de importancia

A saber: en el enfermo

Obediencia y tolerancia:

En los asistentes celo, caridad y vigilancia:

Buen alimento, remedios

De propiedad, buena cama.

Ropa limpia, olores gratos,

Habitación ventilada,

Tranquilidad del espíritu.

$Y$ además que a vencer valgan

la enfermedad, o cooperar

A extinguirla, o moderarla.

Para abordar el tema de la mujer como profesional de la salud es necesario analizar dos aspectos vinculados: la teoría del desarrollo moral femenino de Carol Gilligan y la vocación de la mujer por desarrollarse en las profesiones de la salud.

\section{Fundamentos}

Entre las teorías del desarrollo moral, la de Kohlberg define la adquisición de la conciencia moral como el desarrollo de un sentido individual de justicia. Sin embargo, Gilligan sostiene que las mujeres definen su moralidad como la capacidad de situarse en el punto de vista de la otra persona y como una inclinación a sacrificarse para asegurar el bienestar del otro. Los hombres se desenvuelven entre lo formal y abstracto, en el respeto a los derechos formales de los demás, en el ámbito individual y en las reglas. Las mujeres actúan en lo contextual, en la responsabilidad por los demás, en las relaciones y en una concepción global y no sólo normativa de la moral. Esta diferencia se genera a partir de un esquema de aprendizaje constructivo, sociocultural, vital y moral que recibimos los hombres y las mujeres en la sociedad.

Gilligan elaboró un cuadro del desarrollo moral en el ámbito de la ética del cuidado. Estableció tres niveles de desarrollo que, aunque se estructuran sobre los estadios de Kohlberg, tienen un contenido muy diferente. Esto tiene que ver con las diferencias básicas de género: ambos géneros defienden la igualdad, pero la ética de la justicia pone el acento en la imparcialidad y la universalidad, lo que elimina las diferencias, mientras que la del cuidado pone el acento en el respeto a la diversidad y en la satisfacción de las necesidades del otro.

Desde este punto de vista se entenderá la diferente orientación que la ética del cuidado de Gilligan da al desarrollo moral: 


\begin{tabular}{|l|l|}
\hline Primer nivel & Atención al Yo para asegurar la supervivencia: el cuidado de sí misma. \\
\hline Transición & Consideración del planteamiento del primer nivel como egoísta. \\
\hline Segundo nivel & $\begin{array}{l}\text { Conexión entre el Yo y los otros por medio del concepto de responsabilidad: la aten- } \\
\text { ción a los demás y la relegación de sí misma a un segundo plano. }\end{array}$ \\
\hline Transición & $\begin{array}{l}\text { Análisis del desequilibrio entre autosacrificio y cuidado, reconsideración de la relación } \\
\text { entre el Yo y los otros. }\end{array}$ \\
\hline Tercer nivel & $\begin{array}{l}\text { Inclusión del Yo y de los otros en la responsabilidad del cuidado. Necesidad de equi- } \\
\text { librio entre el poder y el cuidado de sí misma, por una parte, y el cuidado a los demás, } \\
\text { por otra. }\end{array}$ \\
\hline
\end{tabular}

En el quehacer práctico, en el tercer nivel se adquiere seguridad, estabilidad y dignidad. Desempeñar una profesión de la salud es una instancia donde se pueden desarrollar estos valores y hacer que cada mujer contribuya eficazmente en todas las áreas en que participa: personales, familiares, sociales y profesionales.

El trabajo es el campo donde germinan nuestros talentos, nuestra creatividad y originalidad. Es la extensión de la esencia de cada yo: como auténticamente se es; de este modo, la persona se proyecta y da sentido y fin a su vida, más allá de lo alcanzable a corto plazo, más allá de las competencias inútiles o del logro de los fines materiales en exclusividad; éstos, si bien aumentan la capacidad de tener, no incrementan la posibilidad de $s e r$.

Redescubrir el valor del quehacer junto con otros nos hace cocreadores de una actividad que nos lleva más allá de nosotros mismos, transforma el cansancio, la fatiga o la rutina en el placer de compartir y hacernos más personas(2).

\section{Materiales y métodos del informe}

El siguiente es un informe descriptivo e interpretativo, que extrae elementos de la información oficial sobre el grupo de estudiantes mujeres que ingresan regularmente a carreras de la salud en la Facultad de Medicina de la Universidad de Chile.

Se recogen, además, datos extraídos de la entrevista de ingreso en una de las escuelas, predominantemente femenina, y de la evaluación metodológica de otras escuelas con semejantes características. Esta última información se obtiene en el marco de la asignatura de bioética que se ofrece a mediados del currículo de la carrera.

\section{Resultados}

La tabla 1 presenta el número de estudiantes que son seleccionadas mediante el proceso regular de ingreso a las universidades chilenas. Se consideran las matrículas de las escuelas de la salud en la Facultad de Medicina de la Universidad de Chile, pues en ellas es posible evaluar las competencias afectivas en diferentes etapas curriculares.

El registro de ingreso a la casa de estudios corresponde a la promoción del presente año. Los datos sobre desarrollo axiográfico, que se consignan en la tabla 2, corresponden a estudiantes mujeres en proceso de formación. Las evaluaciones se realizan entre primer y quinto grado, en el marco de las asignaturas de ética y bioética que se imparten en tres escuelas profesionales con mayor presencia femenina. 
Tabla 1: Ingresos 2006

\begin{tabular}{|l|c|c|c|}
\hline Escuelas de la salud & Hombres & Mujeres & Total \\
\hline Enfermería & 13 & 72 & 85 \\
\hline Fonoaudiología & 7 & 26 & 33 \\
\hline Kinesiología & 10 & 22 & 32 \\
\hline Medicina & 95 & 65 & 160 \\
\hline Nutrición & 8 & 29 & 37 \\
\hline Obstetricia & 13 & 64 & 77 \\
\hline Tecnología Médica & 28 & 49 & 77 \\
\hline Terapia Ocupacional & 4 & 32 & 36 \\
\hline Totales & 178 & 359 & 537 \\
\hline Porcentajes & $33,2 \%$ & $66,8 \%$ & $100 \%$ \\
\hline
\end{tabular}

Las teorías del desarrollo moral coinciden en dos aspectos; por una parte, establecen que, durante el proceso, se definen motivaciones que norman el comportamiento, es decir, se van formando los valores que guían las interacciones entre los seres humanos. Por otra, visualizan la adolescencia como la etapa crucial en la cual las personas revisan y confirman sus valores.

La elección de una carrera profesional es una decisión que se efectúa precisamente durante la adolescencia. Optar por una profesión es la oportunidad clave para expresar los valores que se han ido generando a lo largo del desarrollo moral.
Desde la base que otorga la literatura disponible sobre este tipo de desarrollo(2,3), ciertas escuelas, al inicio de las actividades académicas, solicitan al estudiante -como parte de sus actividades oficiales- indicar los principales valores que impulsan su vocación. Además, en el marco de las asignaturas de ética o bioética se desarrollan talleres donde se identifican los valores que permanecen desde la elección vocacional y los que se incorporan dentro de las competencias profesionales.

En la tabla 2 se presentan los datos recogidos en las escuelas con mayor presencia femenina -Terapia Ocupacional, Obstetricia y Fonoaudiología- que llevan a cabo las actividades académicas descritas.

Tabla 2: Valores vocacionales

\begin{tabular}{|l|c|c|c|c|c|c|}
\hline Valores & \multicolumn{2}{|c|}{ Entrevista de Ingreso } & \multicolumn{2}{c|}{ A mediados del programa } & \multicolumn{2}{c|}{ Al final del programa } \\
\hline & \multicolumn{2}{|c|}{32 mujeres $^{1}(64)$} & \multicolumn{2}{c|}{38 mujeres $^{2}(108)$} & \multicolumn{2}{c|}{48 mujeres $^{3}(148)$} \\
\hline & $\mathrm{N}^{\mathrm{o}}$ & $\%$ & $\mathrm{~N}^{\mathrm{o}}$ & $\%$ & $\mathrm{~N}^{\mathrm{o}}$ & $\%$ \\
\hline Prudencia & - & & 10 & 9,26 & 26 & 17,57 \\
\hline Perseverancia & - & & 5 & 4,63 & - & \\
\hline Responsabilidad & - & & 4 & 3,70 & - & \\
\hline Justicia & 2 & 3,13 & 16 & 14,82 & 10 & 6,76 \\
\hline
\end{tabular}

1 En la entrevista de ingreso se les pide mencionar dos motivaciones que fundamentan su elección profesional.

2 En el Taller de Bioética, en $4^{\circ}$ año, se les solicita establecer tres valores básicos para alcanzar competencias profesionales.

3 En la Reunión Clínica en Bioética, durante el internado, se jerarquizan lista de 12 valores, de acuerdo con las competencias profesionales. Los resultados indican el número de veces que fueron mencionados en los tres primeros lugares por integrantes de cada grupo de trabajo. 


\begin{tabular}{|l|r|r|r|r|r|r|}
\hline Amistad & 12 & 18,75 & 3 & 2,78 & - & \\
\hline Respeto & - & & 15 & 13,89 & - & \\
\hline Comprensión o empatía & 16 & 25 & 9 & 8,33 & - & \\
\hline Orden & - & & - & & - & \\
\hline Servicio & 10 & 15,63 & 9 & 8,33 & 40 & 27,03 \\
\hline Solidaridad & 6 & 9,38 & 19 & 17,59 & 13 & 8,78 \\
\hline Benevolencia & - & & 9 & 8,33 & - & \\
\hline Autorrealización & 14 & 21,88 & 6 & 5,57 & 51 & 34,46 \\
\hline $\begin{array}{l}\text { Total veces valores } \\
\text { mencionados }\end{array}$ & 60 & & 105 & & 140 & \\
\hline Otros valores indicados & 4 & 6.25 & 3 & 2,78 & 8 & 5,41 \\
\hline
\end{tabular}

\section{Discusión}

La primera tabla nos muestra que aproximadamente dos tercios de los estudiantes que ingresan a las escuelas de las profesiones de la salud son mujeres. Esta relación se ha ido estableciendo en los últimos diez años.

El proceso de formación profesional genera una estructura valórica. Inicialmente, las estudiantes distinguen los resortes emocionales que impulsan la elección vocacional a partir de un "estar en contacto con las personas", "ayudar a lograr el bienestar", "desarrollarse como persona", "aprender las ciencias biológicas". Se destacan los valores de "autorrealización" y "empatía" junto a "servicio y "amistad". Después de completar la formación básica y empezar con las actividades de práctica clínica, los valores se reorientan con mayor fuerza a "prudencia", "justicia" y "respeto en el desarrollo de las funciones profesionales". En esta misma etapa se mantiene la valoración de la "solidaridad". En la fase final de su formación profesional las estudiantes definen como valores capitales para la adquisición de sus competencias la "prudencia", la "justicia" y la "solidaridad"; con claridad se establecen dos pilares fundamentales: el "servicio" y la "autorrealización profesional".

La mujer sigue siendo artífice del mantenimiento de un ambiente social. A ella, que sufrió discriminaciones $-\mathrm{y}$ sufre todavía-, le corresponde, por memoria social y capacidad de ternura, generar un contexto que propicie una genuina posición diversificadora, de defensa, de legítima y directa vinculación, que trascienda la universalidad de la justicia a su vivencia en la individualidad.

El proceso de "ponerse en el lugar del otro" debe ser liderado por la mujer, porque debe partir de un acto de afecto que es el mejor camino para el conocimiento: es allí donde la mujer aporta su punto de vista templado, valorado desde el sentimiento y reflexión serena, subjetiva, y no desde la mera repetición de frases hechas.

Uno de los campos donde la mujer puede realizar este aporte es en los comités de ética. En nuestro país la incorporación femenina ha sido lenta. En el Proyecto de investigación Fondecyt \# 1010947, "Una aproximación histórica a los comités de ética de la investigación en Chile", que desarrolla el Centro Interdisciplinario de Estudios en Bioética de la Universidad de Chile, se menciona que algunas mujeres empezaron a integrarse recientemente para cumplir con necesidades estratégicas del comité. Investigadores e integrantes de comités, que evalúan frecuentemente protocolos, informaron que sólo a partir de 2001 se incorporaron mujeres, particularmente abogadas y jefas de enfermeras. 
Lo anterior evidencia la realidad de muchos ámbitos de desarrollo profesional, en los cuales prevalecen los roles femeninos tradicionales (subordinados), aun cuando se trate de actividades que requieren preferentemente de capacidad de reflexión y pluralidad.

\section{Conclusiones}

La elección profesional es una opción personal de vida que refleja la valoración de una actividad, pues allí se define un modo activo y dinámico de asumir un lugar en la historia de la comunidad y una autocomprensión de sí mismo.
Los progresos de las ciencias de la salud se han traducido en tecnologías; sin embargo, la figura femenina al interior de las profesiones de la salud plantea una tesis humanizante, que conlleva una construcción del hacer coherente con la justicia, la solidaridad y la responsabilidad, y el respeto a la dignidad humana.

La profesión es una forma de realizar la cercanía al legítimo otro en respeto y solidaridad, de reconocerlo en su necesidad y de acogerlo y encontrarlo como un tú. Se trata, entonces, de alcanzar, por medio de la profesión, el desarrollo de una condición racional del sersaber-hacer.

\section{Referencias}

1. García Gual C, (ed.) Tratados Hipocráticos. Vol. I. Madrid: Gredos; 1983: 238.

2. Bravo N. Valores humanos: por la senda de una ética cotidiana. Santiago de Chile: RIL ediciones; 2001

3. Isaac D. La educación de las virtudes humanas. Madrid: Ediciones E.U.N.S.A; 1981.

\section{Bibliografía}

Aranguren JL. Moral de la vida cotidiana, personal y religiosa. Madrid: Tecnos; 1987.

Cortina A. La ética de la sociedad. Madrid: Alianza; 1995.

García G. La noción de profesión, su ser y su sentido: referentes ético-morales en problemas de ética aplicada. Colombia: Ediciones El Bosque; 1987.

Gilligan C. In a different voice: Psychological theory and women's Moral Development. Cambridge: Harvard University Press; 1982.

Gilligan C. La moral y la teoría: psicología del desarrollo femenino. México: FCE; 1985.

Habermas J. Conciencia moral y acción comunicativa. Barcelona: Península; 1985.

Kohlberg L. El enfoque cognitivo-evolutivo de la educación moral. En; Santolaria F, Jordán JA, (comp.) La educación moral, hoy. Cuestiones y perspectivas. Barcelona: PPU; 1987.

Kohlberg L, Power FC, Higgins A. La educación moral según Lawrence Kohlberg. Barcelona: Gedisa; 1997.

Kohlberg L. Psicología del desarrollo moral. Bilbao: Desclée de Brouwer; 1992.

Laín Entralgo P. Teoría y realidad del otro. Madrid: Alianza; 1982. 
Lolas F. Aspectos éticos de la investigación biomédica. Conceptos frecuentes en las normas escritas. Revista Médica de Chile 2001; 129: 680-84.

Papalia D, Wendkos S. Desarrollo Humano. Bogotá: McGraw-Hill/Interamericana; 1994.

Peters RS. Desarrollo moral y educación moral. México: FCE; 1984.

Recibido el 27 de abril de 2006.

Aceptado el 04 de mayo de 2006. 